\title{
Review of: "Diagnostic value of F-18 FDG PET/CT in fever or inflammation of unknown origin in a large single-center retrospective study"
}

JR Garcia

Potential competing interests: The author(s) declared that no potential competing interests exist.

There is a large serie, well written and with results and conclusion according to the literature, but there is not a new issue and is a retrospective an single center study 